\title{
Personalia
}

medgen 2019·31:24

https://doi.org/10.1007/s11825-019-0237-3

Online publiziert: 30. Januar 2019

๑ Springer Medizin Verlag GmbH, ein Teil von Springer Nature 2019

\section{Geburtstag von Professor Alan Eglin Heathcote Emery}

Am 21. August 2018 feierte Alan Emery seinen 90. Geburtstag. Den älteren Humangenetikern ist er bestimmt noch sehr gut in Erinnerung. Er war in drei Bereichen der Humangenetik ein führender Wissenschaftler:

1. Muskelerkrankungen (z. B. Erstbeschreiber der Emery-DreifussMuskeldystrophie, 1966 [1], oder sein Buch über Duchennesche Muskeldystrophie, 1987 [2])

2. Formale Genetik (z. B. sein Buch: Methodology in Medical Genetics, 1976 [3])

3. Klinische Genetik (z. B. das Standard-Werk: Emery, Rimoin: Principles and Practice of Medical Genetics, 1983 [4]).

An der Universität Manchester studierte er Botanik und Zoologie. 1952 schloss er sein Studium mit Auszeichnungen ab. 1956/57 war er einer der ersten ausländischen Gaststudenten an der Medizinischen Fakultät Würzburg. Im Jahr 1960 beendete er sein Medizinstudium ebenfalls an der Universität in Manchester. Anschließend promovierte er in Humangenetik an der Johns Hopkins University in Baltimore (USA). In Manchester arbeitete er später in der genetischen Beratung. Von 1968 bis 1983 war Emery Professor und Vorsitzender der Abteilung für Humangenetik der Universität Edinburgh. Emerys Forschung konzentrierte sich auf neuromuskuläre Erkrankungen und formale Genetik. Er hat mehr als 200 wissenschaftliche Arbeiten veröffentlicht und ist Autor vieler Bücher.

Er war 1992 Mitbegründer des European Neuromuscular Center (ENMC), eine Einrichtung europäischer Selbsthilfegruppen, um ein Netzwerk führender Wissenschaftler und Kliniker zu bilden. Emery war mehrere Jahre Research Director des ENMC.

Emery erhielt zahlreiche Auszeichnungen, darunter den National Foundation March of Dimes International Award for Research und 1995 den Ehrendoktor der Medizinischen Fakultät der Universität Würzburg [5].

Von 1980 bis 1983 war er Präsident der British Clinical Genetics Society. Er war wissenschaftlicher Mitarbeiter und ab 1985 Ehrenstipendiat des Green Templeton College in Oxford. Von 2001 bis 2004 leitete er als erster Präsident die Royal Society of Medicine Section of Medical Genetics. Viele wissenschaftliche Einrichtungen wählten Emery in ihre wissenschaftlichen Gremien:

Fellow des Royal College of Physicians (FRCP), Fellow des Royal College of Physicians von Edinburgh (FRCPE), Fellow des American College of Medical Genetics (FACMG), Fellow der Linnean Society (FLS), Fellow der Royal Society of Arts (FRSA) und ein Fellow der Royal Society of Edinburgh (FRSE).

Mit Sicherheit ist diese Liste der wissenschaftlichen Leistungen und Ehrungen von Emery nicht vollständig. Seit einigen Jahren lebt Emery mit seiner Frau Marcia in Devon. Wir hoffen, dass er noch viele gesunde Jahre vor sich hat.

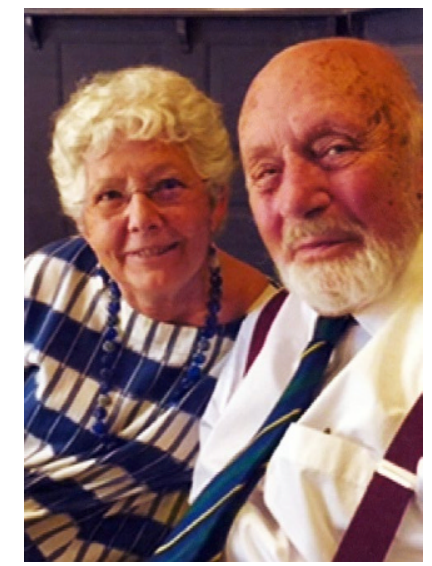

\section{Literatur}

1. Emery AE, Dreifuss FE (1966) Unusual type of benign x-linked muscular dystrophy. J Neurol Neurosurg Psychiatry 29(4):338-342

2. Emery AEH, Muntoni F, Quinlivan RCM (1987) Duchenne Muscular Dystrophy. Oxford Monographs on Medical Genetics, 4. Aufl. Univ Pr, Oxford

3. Emery AEH (1976) Methodology in Medical Genetics: An Introduction to Statistical Methods. Churchill Livingstone, Edinburgh

4. Emery AEH, Rimoin DL (1983) Principles and Practice of Medical Genetics. Churchill Livingstone, Edinburgh

5. Grimm T (1995) Ehrenpromotion für Professor Alan Eglin Heathcote Emery. Med Genet 7:426 